\title{
Radio Anomalies, Acoustic Emissions and Gravitational Variations in the Teaching of Seismology at Secondary School
}

\author{
Valentino Straser \\ Independent Researcher, Strada dei Laghi 8, Terenzo 43040, Italy
}

\begin{abstract}
Following crustal stress and the tectonic evolutions that lead to the triggering of seisms is still premature, for technological reasons. Instead, in view of the energies involved, which are in the order of kilotons, it is necessary to collect symptoms manifesting inside the Earth. The greater the stresses produced, the more evident will be the seismic signals manifesting on a global scale. From the point of view of teaching, it is proposed to study seismology in secondary schools using an "evidential" paradigm, rather than the "Galileian" sort. This will require a more modern approach, one that considers non-linearity an investigation model that is more in line with the Natural Science approach. To this effect, also the seismology lab is transformed from a place where reality is "reproduced", into a setting where comparisons are made in the intrinsic presence of clues rather than proofs. The instruments used to carry out this project, which is taking its first steps in an experimental form in Parma (Italy), can be reproduced at low cost, but without forsaking precision measurements. The instruments in question are those used to detect radio anomalies, acoustic emissions produced in the deepest layers of the terrestrial crust, and variations in gravity that require a computer to interface data and elaborate signals $24 / 7$.
\end{abstract}

Key words: Radio anomalies, gravimetric measurements, acoustic emission, evidential paradigm, Galileian paradigm, seismology.

\section{Introduction}

Seismology is intimately interwoven with other disciplines such as the earth sciences and branches of geophysics: from astronomy to geodesy, and from geology to physics. In just a few centuries, seismology has been transformed into a science which, in addition to pursuing a predictive goal, has cast new light on the internal constitution of the Earth and its properties and physical conditions. Amongst the goals pursued by seismology are those of achieving a profound description of the phenomena from a general point of view and interpreting the causes that produce them.

Unlike other scientific spheres, in seismology observational data prevail over experimental data. In fact it is currently difficult, if not practically impossible, to reproduce in a lab the natural phenomena of interest to seismology at the same

Corresponding author: Valentino Straser, Dr., professor, research field: earthquake prediction. spatial and temporal scale that they manifest in. Compared to what happens in the real world, in these intrinsically chaotic and changing natural phenomena, many environmental elements are involved that are not always known or repeatable in the lab. Consequently, to study seismology, as in other scientific spheres, it is necessary to interpret certain properties or phenomena of the Earth by means of theoretical models that are not always built from direct observation.

Because of the energies involved, the seismology sphere cannot simply be circumscribed at a local level but must take into account a global vision of the phenomena, considered with an interdisciplinary context.

In fact, crustal stresses act on a planetary scale and propagate through the Earth's crust to generate "crustal storms", alternating with periods of seismic calm. Earthquakes, above all those where significant 
amounts of energy in the order of kilotons are released, occur during periods of "crustal storms", and strike only where some fault has accumulated sufficient elastic energy to generate a seismic shock [1-3]. Because of the energies involved, it is therefore possible to follow, also instrumentally, the propagation of the endogenous tensions that manifest short term on a temporal scale. The study of crustal stresses, in a global vision, cannot be made "only" by using seismometers, which indicate the conclusive moments of a long geophysical process when a seism has already occurred.

To overcome this obstacle, it is necessary to find diagnostic tools that can follow the state of the crust as it evolves even when an earthquake does not occur, which does not exclude the interaction of the Earth with planetary dynamics and solar activity.

Subscribing to a concept of non-linearity and "chaotic" patterns that preside over both natural and cosmic phenomena, every earthquake is a "unique" phenomenon not comparable with other seismic events.

The changes in the release of endogenous energy are huge from year to year and can be followed via a crust diagnosis, also using "scaled-down" instrumentation, at low cost, in the order of hundreds of dollars, to be used in secondary school labs.

With current technology, it is virtually impossible to monitor the evolution of the stresses produced in a fault. Instead, it is possible to follow the endogenous tensions propagated globally via detection of signals of an electrical and electromagnetic variety, generated by minerals subjected to tectonic stresses [4-12]. In particular, both in terms of the costs and reliability of the 24/7 detection method, it has proved particularly effective to monitor radio emissions in a frequency from $0-3 \mathrm{~Hz}$ that precede and follow potentially destructive seismic events with a magnitude equal to or greater than M6 on the Richter scale [13].

Further information on the evolution of endogenous stresses can be obtained by monitoring the Earth's gravity using a pendulum gravimeter, this too homemade, but precise to the eighth decimal place.

The Moon, with its tidal force, changing with depth inside the Earth, is the first engine of the terrestrial dynamo which manifests in a tiny measurement as the Earth's magnetic field, and to a great extent as heat released by the deep electrical currents that are the primary origin of the Earth's endogenous heat, and its temporal variations. The available energy involved is enough on its own to justify all the phenomena of endogenous origin, including climatic variations. The thermal expansion of the Earth's deepest layers is responsible for geodynamic phenomena, earthquakes and volcanic eruptions [14].

A further element to be monitored, again $24 / 7$, is solar activity.

The electromagnetic phenomena linked to the solar wind and its variations clearly influence the terrestrial dynamo and the generation of endogenous heat, and hence the terrestrial dynamics. Therefore, there are certainly links between the variation in cyclic solar activity with terrestrial seismicity, even for a particular solar phenomenon that can be decisive [15-18]. The monitoring and forecasting of solar activity can be followed directly on the website.

Last but not least, and again at low cost, in the order of tens of dollars, it is possible to monitor "crustal storms" using acoustic sensors which, though of low quality, can indicate seismic events of both a local and global nature, of a particular intensity.

Using a PC, the instrumentation can be set up in a school lab. The synergy created between the measurements and detection methods suggested in this study, enhanced with constant information on the seismic events occurring worldwide, that can be consulted on the website, provide an overview of the progress and evolution of the ongoing endogenous tensions.

The instrumentation, tested for over 8 years, is operational 24/7 at Rovigo (measurement of gravity and acoustic anomalies), Rome (detection of 
geomagnetic background and radio anomalies) and Parma where a seismology lab has been set up for teaching purposes. The project looks at the three types of signals measured: electromagnetic, acoustic and gravitational [1].

\section{A Question of Method}

The laboratory concept is closely linked to the Galileian paradigm, which brings together observation, hypothesis, experimentation and mathematical calculation. In practice, the scientific method proposes to obtain information on the mechanism of natural events, formulating answers to find out whether the solutions proposed are valid. It follows that science based on the Galileian paradigm proposes to achieve predictive abilities when it comes to a particular natural phenomenon. For disciplines such as seismology, in view of the energies involved, in the order of Kilotons or Megatons, it becomes difficult, if not impossible, to reproduce the mechanics of an earthquake on a real scale. In this sphere, the observer interacts with the final result, often based on the clue to an effect that can neither be experienced nor directly observed. Over the last few decades, with the introduction of theories such as The Big Bang and Black Holes, which seem to have little to do with Galileian experiments, the spatial-temporal confines of nature have expanded remarkably. Earthquakes are part of those phenomena that cannot be directly observed because of technological limits. Astrophysics and cosmology, founded on the General Theory of Relativity, are sciences, forms of knowledge that, though not excluding description, forecasting, mathematical language and the idea of totality, must nonetheless measure themselves against the intrinsic possibility of carrying out experiments. To overcome this impasse in scientific investigation, the term "evidential paradigm" has been coined, which has proved to be of use when describing natural phenomena that cannot be simulated in the lab. In other words (for Ginzburg) this is a way of using experiential data to arrive at a complex reality that cannot be experienced directly.

A new way of working that modifies the relationship between man and nature, where, according to Rosen and Hawking, "the subject knows the object but cannot dominate it because it will not allow itself to be reduced to an object that is reproducible, decomposable and controllable in the laboratory".

On the contrary, in the Galileian paradigm, predictability is the predictive power of the theory and its objectivity, guaranteed by the reproducibility of the results. It is in this context that the Fab Lab concept expresses the invention of the reproducible. In a certain sense, the "evidential paradigm" represents the opposite of an exact science, i.e., where comparisons should be made with the intrinsic presence of clues and not proofs.

In this particular project, this means adopting an attitude of curiosity towards seismology, i.e. following the symptoms that the Earth communicates to us through electromagnetic, gravitational and acoustic signals, when crustal stress is building up and evolving. In other words, when the tension of the rocks is already close to breaking point, or to an elastic recovery, to evolve into an earthquake. This "elastic" way to approach a complex science like seismology, can find points in common with the Galilean paradigm, whose strength is based on its predictive power, while the evidential kind is characterized by the non-reproducibility of phenomena involving high levels of energy. Nonetheless, the evidential method makes it possible to come close, from a conceptual point of view, to complex, more modern phenomena, such as "non-linearity" and "deterministic chaos".

\section{Instruments}

\subsection{Measurements of Electromagnetic Waves}

The receiver set for continuous bandwidth reception (VLF $0 \mathrm{~Hz}-30 \mathrm{kHz}$ ), was connected to a 1.5 metre antenna and an ARGO Data Acquisition System. 
The "SELF/LF Amplifier" is a USB-powered portable radio receiver, designed for the "Natural Radio" study [2]. Designed to be used in the field for short periods (a few hours) or to work continuously when environmental electromagnetic monitoring lasting some weeks or months is required. The receiver's enclosure meets the IP55 standard (high resistance to continuous water jets and dust) which makes it possible to operate in adverse meteorological conditions as well as environments featuring large quantities of moisture/dust (e.g. in contact with soil). These characteristics make it particularly suitable for uses in the fields of geophysics, geology and ham radio. The heart of the receiver is an OP37GP precision, high speed operational amplifier which boasts extremely low electronic noise levels (circa 80 $n V$ p-p $0.1 \mathrm{~Hz}$ to $10 \mathrm{~Hz}$ ) and an offset voltage that never exceeds $100 \mu \mathrm{V}$. This Chip can amplify both electrical signals with constant voltage (DC or $0 \mathrm{~Hz}$ ), and $\mathrm{AC}$ signals with a maximum of $63 \mathrm{MHz}$ (VHF band). In other words, the OP37GP can amplify radio signals within a bandwidth of $63 \mathrm{MHz}$ : a value much higher than the bandwidth normally associated with natural radio $(0-100 \mathrm{kHz})$, i.e. those radio signals generated by the movement of electrical charges present in the troposphere/ionosphere or produced by the interaction between the solar wind and the Earth's magnetosphere.

\subsection{Software}

"Spectrum Lab" set as follows:

Effect of FFT settings with FS $=22.0500 \mathrm{kHz}$ :

Width of one FFT-bin: $21.0285 \mathrm{MHz}$

Equiv. noise bandwidth: $28.5988 \mathrm{MHz}$

Max frequency range: $\mathrm{Hz}-1378.13 \mathrm{~Hz}$

Data collection for one new FFT: $47.554 \mathrm{~s}$

Overlap from scroll interval: $97.9 \%$

Resolution: $0.02 \mathrm{~Hz}$

Registration field: 1 line/s (500 ms)

Antenna pointing towards the nadir.

\subsection{Gravimetric Measurements}

The gravimeter has a device which is independent from barometric pressure variations and a pendulum with low expansion rods to limit errors due to thermal expansion. The oscillator with a position finder, which can produce a very precise synchronism signal, has no electromagnetic interference and is connected to an electronic clock which is precise to the eighth-ninth significant digit. This system is controlled by a calculator. In one day, about 52 values of the Earth's gravitational field are obtained and data continue to be collected between one measurement and the next thanks to being recorded on a disk. The relative error over 1,000 measurements is 0.000000089 .

\subsection{Acoustic Emission}

The device used for the instrumentation is normally employed in 0-40 Khz ultrasonic alarm systems, which detect infra/ultrasound portions. In Italy, this probe costs around two dollars, and can be bought from electronics stores. It is connected using a cable for audio or satellite systems fitted with standard mono audio jacks, insulated by a PVC or corrugated tube for brickwork. To monitor acoustic emissions a screen can be downloaded from internet with the free software called SPECTRAN, or alternatively SPECTRUMLAB, again free on internet.

The device can be lodged in a small laboratory or shelter, protected from bad weather, connecting its probe to the PC's audio card via the mike input.

3.5 Procedure to Measure the Geomagnetic Field (Dr. Gabriele Daniele Cataldi Method, LTPA Radio Emission Project)

The procedure to monitor the geomagnetic field employed by the authors to carry out a correlation study used analogue radio receivers featuring ultra-low-noise high-speed precision operational-amplifiers that operate efficiently in the following bands: SELF ( $<3 \mathrm{~Hz})$, ELF (3-30 Hz), SLF (30-300 Hz), ULF (300-3,000 Hz), VLF (3-30 kHz) 
and LF (30-300 kHz) via wire-loop antennae and antennae sensitive to magnetic fields (bobbins) aligned with the vectorial components of the geomagnetic field.

The radio signals collected, after being suitably amplified, are sent to a PC that converts them into real-time digital signals and analyses their spectrometric characteristics (frequency and intensity) to produce spectrograms by means of FFT software (software that uses Fast Fourier Transform).

All the amplification systems (radio receivers) the monitoring station is equipped with, including the antennae, are prototypes designed and built by Gabriele Cataldi. The main monitoring system is a prototype SELF/ELF radio receiver featuring a bobbin antenna consisting of three multilayer coils wrapped around a ferromagnetic core and connected in series (a magnetic induction antenna) for a total of $468.4 \mathrm{k}$ turns. The antenna is aligned vertically (parallel to the $\mathrm{Z}$ component of the geomagnetic field).

\subsection{Characteristics of Seismic Geomagnetic Precursors or SGPs (Seismic Geomagnetic Precursors)}

Seismic Geomagnetic Precursors or SGPs are variations in the Earth's geomagnetic field. These are geomagnetic variations associated with a variation in solar activity that precede strong earthquakes with a magnitude of at least $6 \mathrm{Mw}$ or M6+. The data from monitoring the SELF-ELF band show that the spectrographic characteristics of these radio emissions are those typical of a geomagnetic perturbation following an increase in solar activity and appear as general increases in the Earth's geomagnetic field at a frequency between $<3 \mathrm{~Hz}$ and $\sim 10-15 \mathrm{~Hz}$, with an intensity directly proportional to their wavelength.

Taking as a reference the peak of the electromagnetic anomaly recorded (SGP), it became possible to calculate the temporal difference between this and the M6+ seism: the average temporal difference recorded was $\sim 598$ minutes ( $\sim 9$ hours). The minimum temporal difference recorded was 1 minute (M6.4 Balleny Islands earthquake, 9 October 2012); the maximum temporal difference recorded was 2,241 minutes (M6.0 Kuril Islands earthquake, 9 September 2012). The distribution of the time intervals tends to diminish in relation to the increase in seism magnitude.

After analyzing the spectrographic characteristics of the SGPs the LTPA researchers found that $6 \%$ of the M6+ earthquakes occurred while the geomagnetic background was still increasing. A good $9.9 \%$ of the earthquakes occurred during the first maximum reduction in the geomagnetic background increase (the authors called this the "Normalization Point" or NP). Normalization points are the moment when the intensity of the geomagnetic background returns to a base or quiescent level. The remaining $84.1 \%$ of earthquakes occurred after the disappearance of the geomagnetic anomaly.

\subsection{Procedure to Measure the Gravity (Dr. Mario Campion)}

The gravity variations associated with earthquakes have been observed by different authors, both with instruments placed in the monitoring station and by satellite [19-22].

The instrument used in this case for monitoring the gravitational field (Rovigo, Italy-coordinates: Latitude $+45.07 \mathrm{~N}$ and Longitude $-11.778 \mathrm{E}$ ) differs significantly from those normally used, which are essentially of three types:

- the first is a spring gravimeter with constant length;

- the second is able to measure the absolute gravity, also known as free-fall gravimeter;

- and the third one which works with the sensing element electromagnetic balance.

The instrument created by Dr. Campion differs significantly from these three types, and may have advantages in terms of measurement compared to them. 
It basically refers to a gravimeter which makes the most of the potential of the simple pendulum, as long as its functionality is optimized with advanced technologies.

The tool measures the average value of gravity in a defined time interval, by timing with extreme precision the time that the oscillator needs to perform 1,000 or 100 or 10 oscillations, then dividing the value by the number of oscillations itself.

When determining the extent of $\mathrm{g}$ with 1,000 oscillations, the figure is averaged over nearly half an hour, and at the end of the day, a graph is created with about 50 intervals on the abscissa and 50 on the ordinate, with related values.

The variations of the average period are inversely proportional to the variations of gravity $g$, in the range within which the instrument carried out the measurement, as resulting from the formula of the simple pendulum.

When working with 100 or 10 oscillations, the measures are obtained by intervals ten or hundred times shorter, so that the analysis of the phenomena is very detailed.

According to Dr. Campion, another great advantage of this instrument is that it is able to sum all the gravity values that have acted on the oscillator, one after the other.

So that the entire trend of the force of gravity, as detected by the oscillator, comes into the evaluation of its average value, also performing a compression of the data, whose limited amount can be easily used.

Considering the 100 measures, the starting interval begins to be perceivable, but in any case it will only take up about thirty percent of the overall interval.

Considering the 10 measures, the measuring interval is reduced to ten percent, and in any case much wider than other measuring systems of $g$.

To complete the description of the device, consider that it is controlled by a computer which manages the operating cycle automatically.

The graphs drawn on a daily basis have changes in the average period as an index of $g$, and are much larger than the considered values of gravity measured by spring or free-fall gravimeters.

In our case, the period may vary from 5 or 6 millionths, and these variations are much larger than the values of $g$ measured with other gravimeters.

This amplification could be the result of this measurement method, which concerns the amount of repeated values, and also the consequence of the use of a sensor with an angular horizontal momentum, which is perpendicular to both the plane of oscillation and the direction of $g$.

The graphs do not show daily and on a regular basis the curve of tidal forces, but over a complete cycle, they point out two significant events which clearly interpret the tidal forces, drawing graphs of similarity with those of the tidal forces, always with great amplification of the phenomenon.

\subsection{Procedure to Monitor Acoustic Emissions (Dr Jerry Ercolini Method)}

The device used, both for simplicity and low cost is easy to position, buried at a depth of least $50 \mathrm{~cm}$ near the computer. Checks made in the immediate vicinity of the monitoring station, also using artificial percussion, demonstrated that the instrument is independent of sound sources of an anthropic type.

\section{Monitoring Examples}

\subsection{Variation in Gravity during the Lunar Phase}

From daily analysis of the graphs we can infer that during the lunar month there are two critical moments, one closer to the new Moon (generally more pronounced) and one closer to the full Moon, in which the trend of gravity recorded by the gravimeter agrees with tide prediction for Italy's Adriatic Sea.

Fig. 3a shows the gravity pattern on February 2, 2011, the day before the new Moon. The recording was carried out with the gravimeter set to 100 measures at 4-minute intervals.

The trend curve (black marker) of the average 

in the Teaching of Seismology at Secondary School

measured data mirrors the trend of the tides in the Adriatic Sea, the maximum corresponding to the passages of the Sun and the Moon over the meridian.

Comparing this trend with the graphs of tidal forces measured using other gravimeters, will show the amplification of tidal forces highlighted by this type of gravimeter: the variation between maximum and minimum of the tide, which emerges from the graph, is 5.5 millionths of $\mathrm{g}$.

4.1.1 Variation in Gravity during the Japanese Earthquake of 11 March 2011

During this catastrophic earthquake in Japan, the gravimeter recorded the event with low-frequency signals which are open to important interpretations (Fig. 3b).

The unit was operating with 10 measures on 10 oscillations and, therefore, obtaining average gravity values every 15 seconds as part of 135 -second intervals.

Therefore, analysis of the event was detailed, namely, using a total of 650 daily measurements.

The graph shows the registration of a 6-hour interval, and follows the event in detail with continuous recording from 6 a.m. to 2 minutes before 12 a.m.

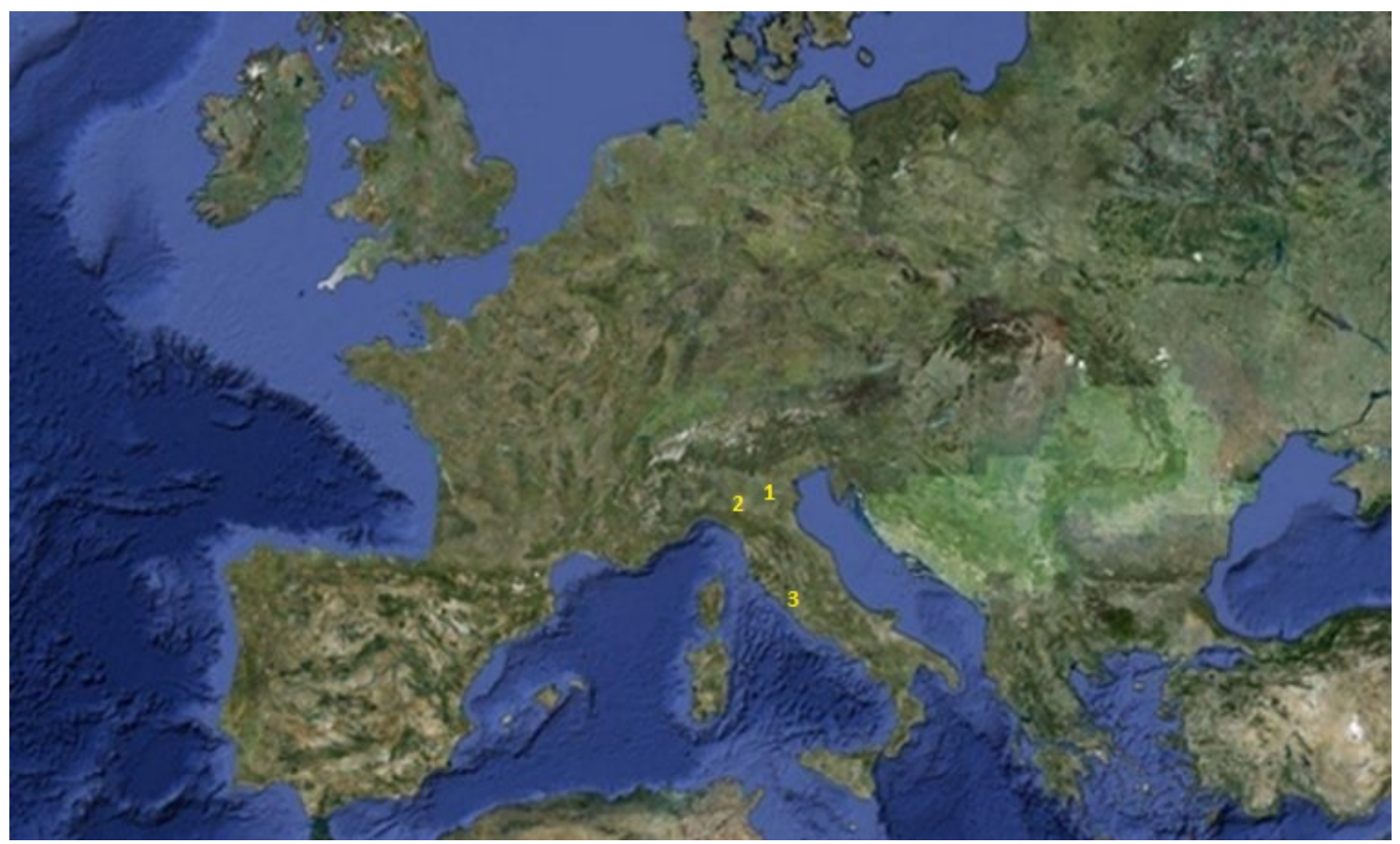

Fig. 1 Index map. (1) Rovigo-experimentation with acoustic emissions and gravimetric anomalies. (2) Parma-site of the experimental educational method. (3) Rome, detection of electromagnetic background and radio anomalies.

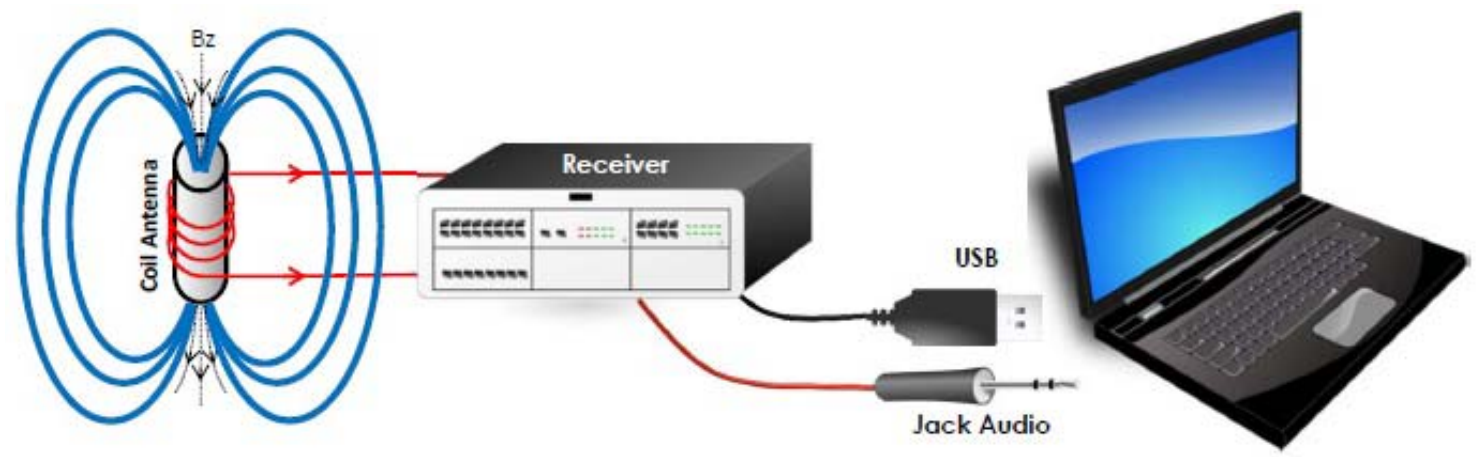

Fig. 2 Scheme of the method to measure the geomagnetic background created by Gabriele and Daniele Cataldi of the Radio Emissions Project observatory in Rome. 
Radio Anomalies, Acoustic Emissions and Gravitational Variations in the Teaching of Seismology at Secondary School

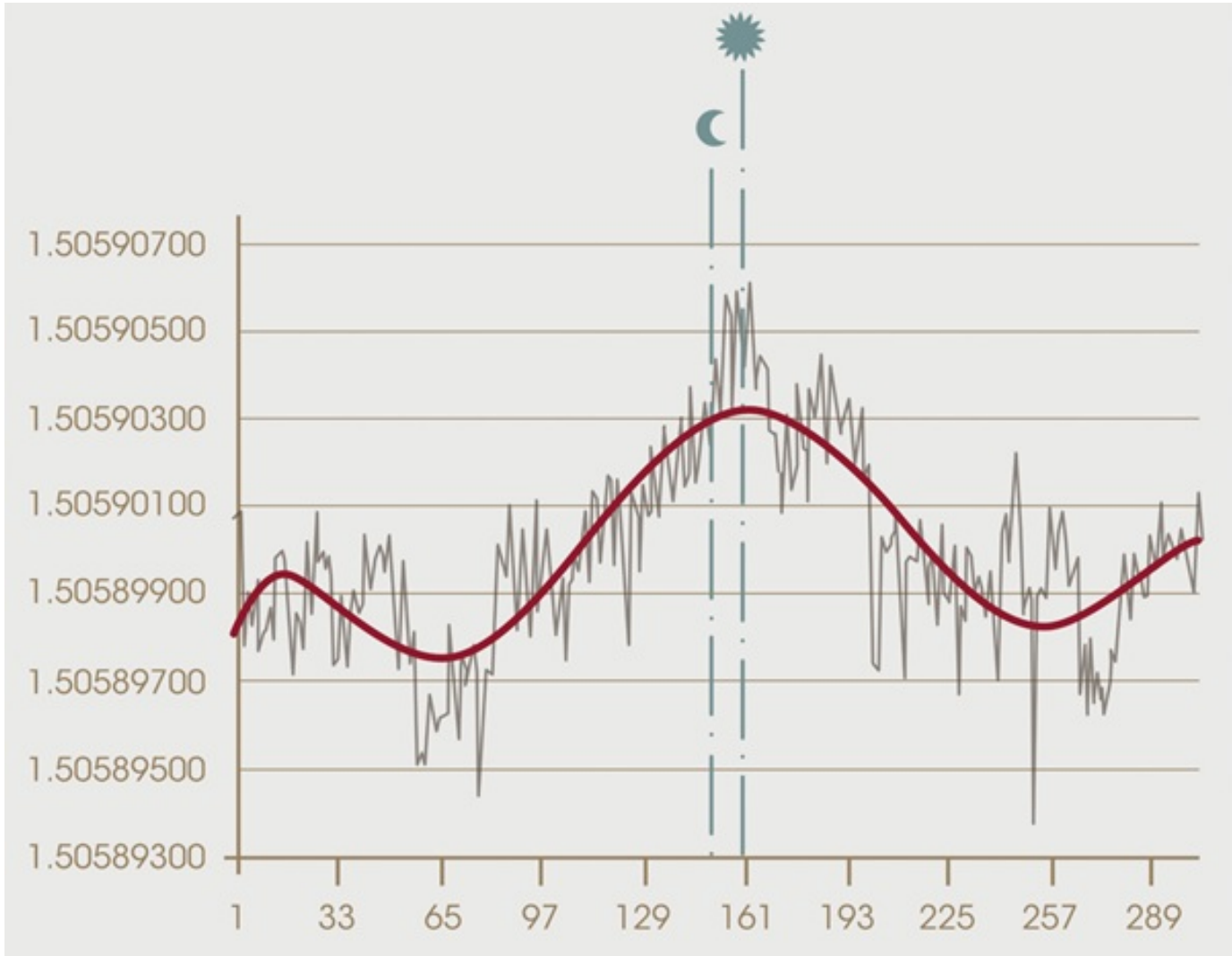

(a)

March 11,2011

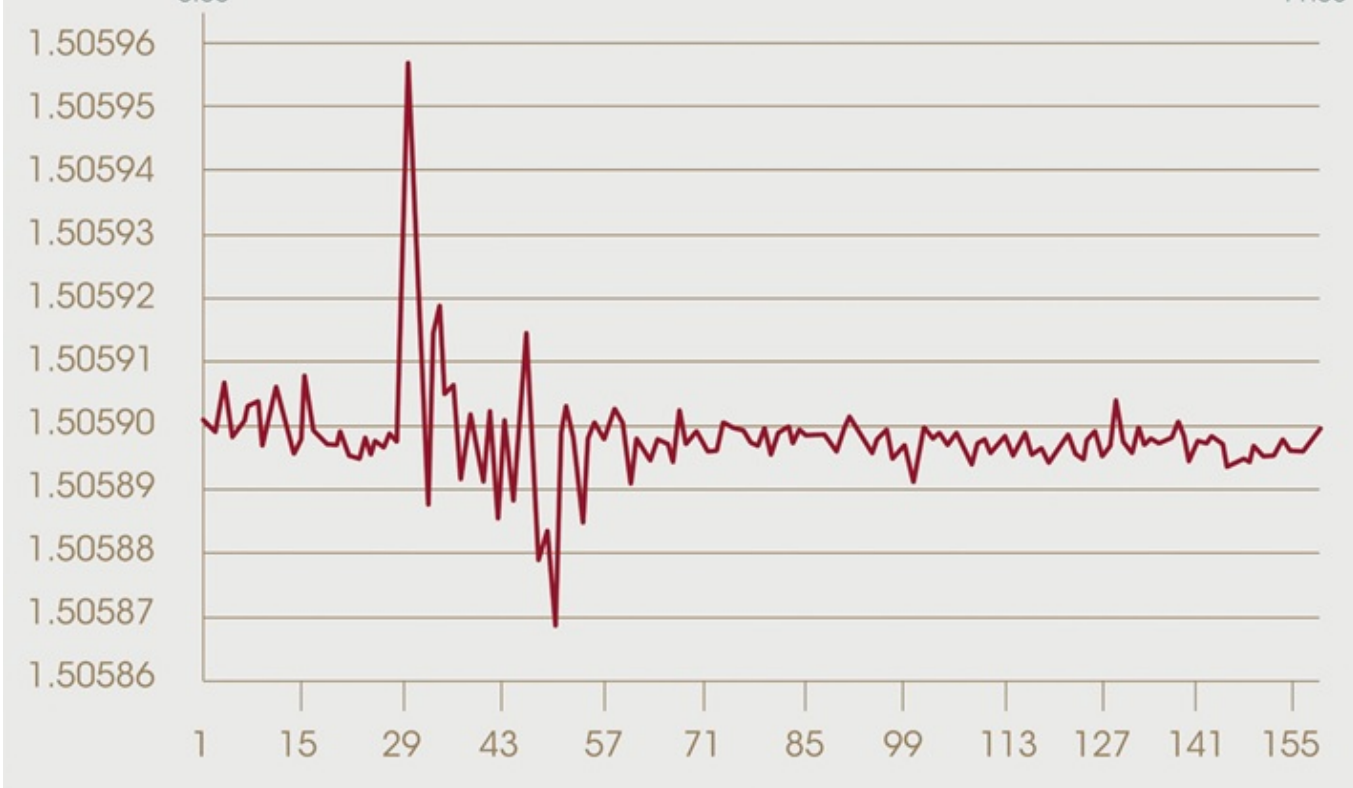

(b)

Fig. 3 (a) Gravity patterns on February 2, 2011, the day before the new Moon, at the passage to the meridian, measured near Rovigo (Italy) by Dr. Mario Campion using a gravimeter of his own design. (b) Gravity pattern during the catastrophic Japanese earthquake of 11 March 2011, which occurred when the increasing gravity reached a peak. 

in the Teaching of Seismology at Secondary School

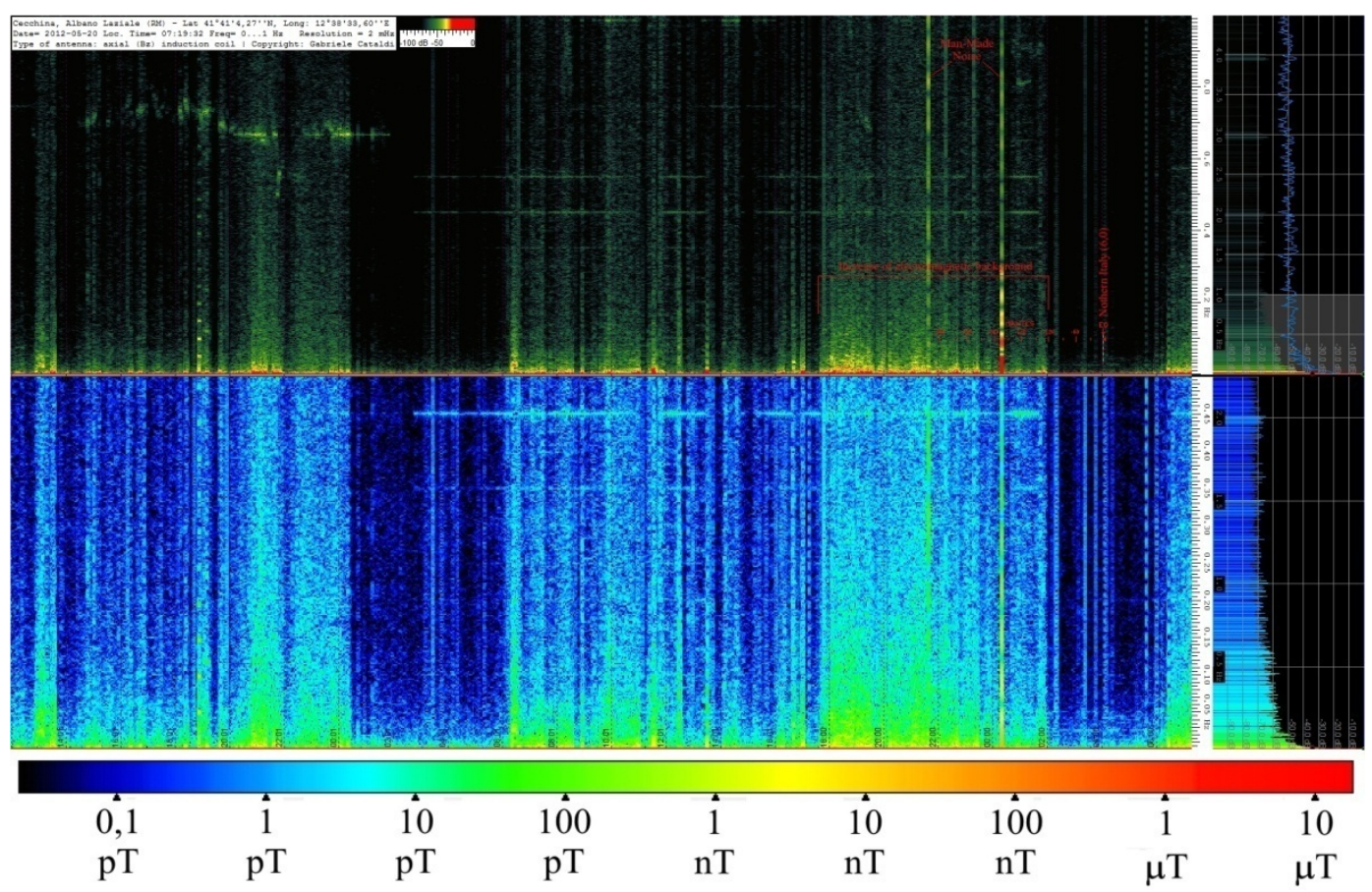

(a)

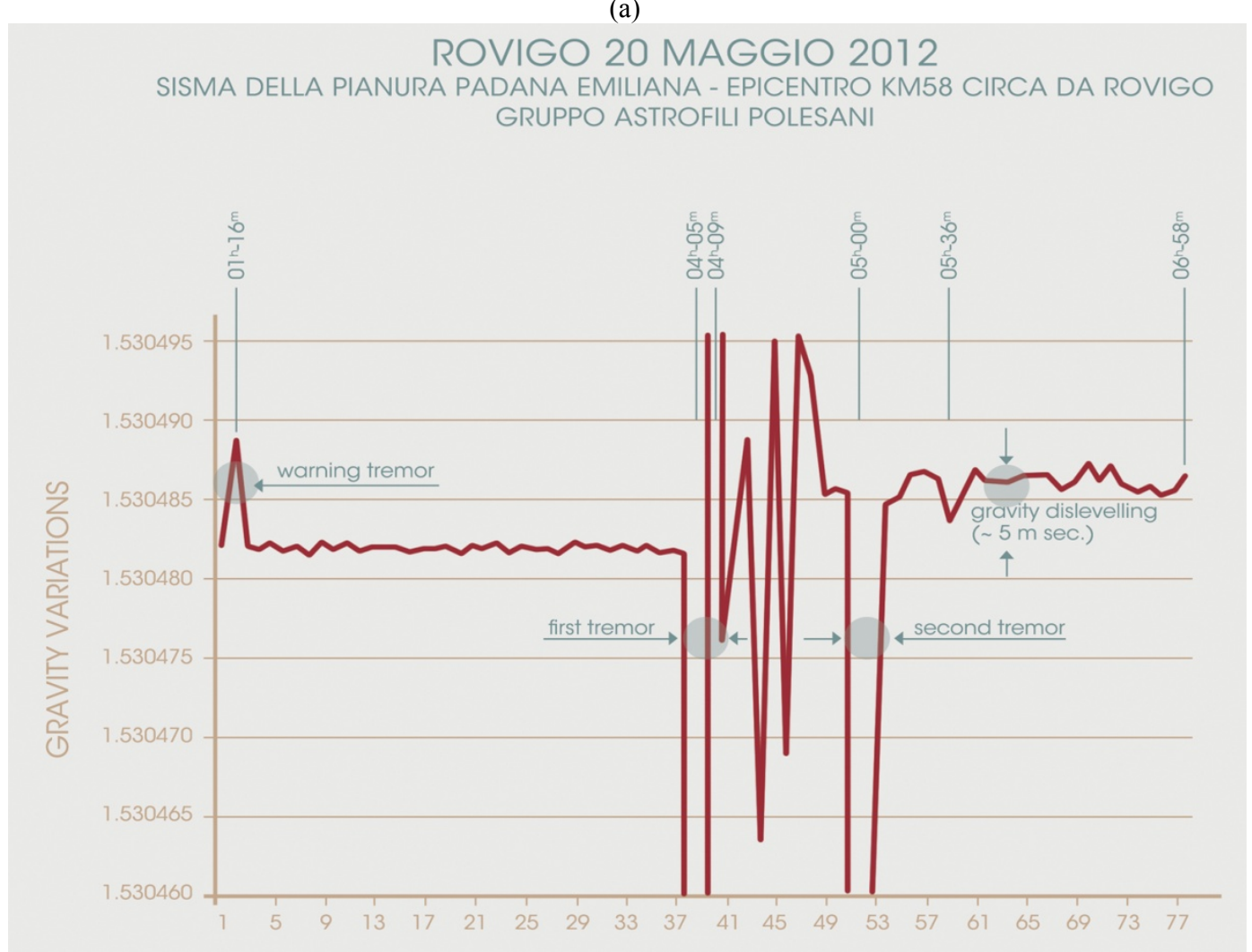

(b)

Fig. 4 Radio and gravimetric anomalies in the Po Valley Plain on 20 May 2012.

Geomagnetic background changes with the presence of radio anomalies, measured by the Radio Emissions Project observatory in Rome, before, during and after the earthquake of 20 May 2012 in the Po Valley Plain. In the graph can be seen the increase in the geomagnetic background preceding the mainshock, which occurred when the electromagnetic signal had "normalized".

The strong fall in the already diminishing gravity can be seen during the mainshock. This fall may have been further accentuated by the shaking of the ground during the triggering of the seism. 
Radio Anomalies, Acoustic Emissions and Gravitational Variations in the Teaching of Seismology at Secondary School

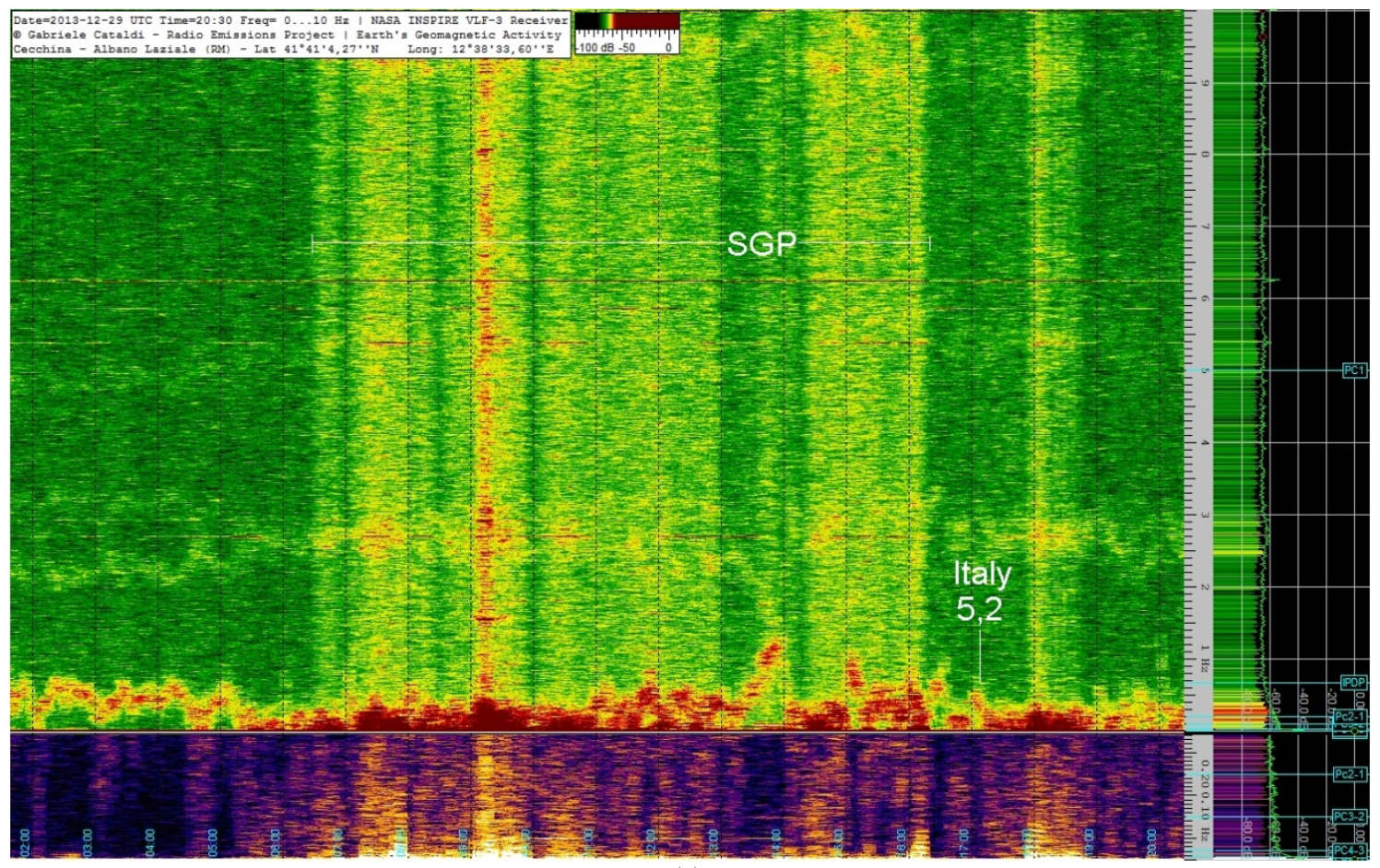

(a)

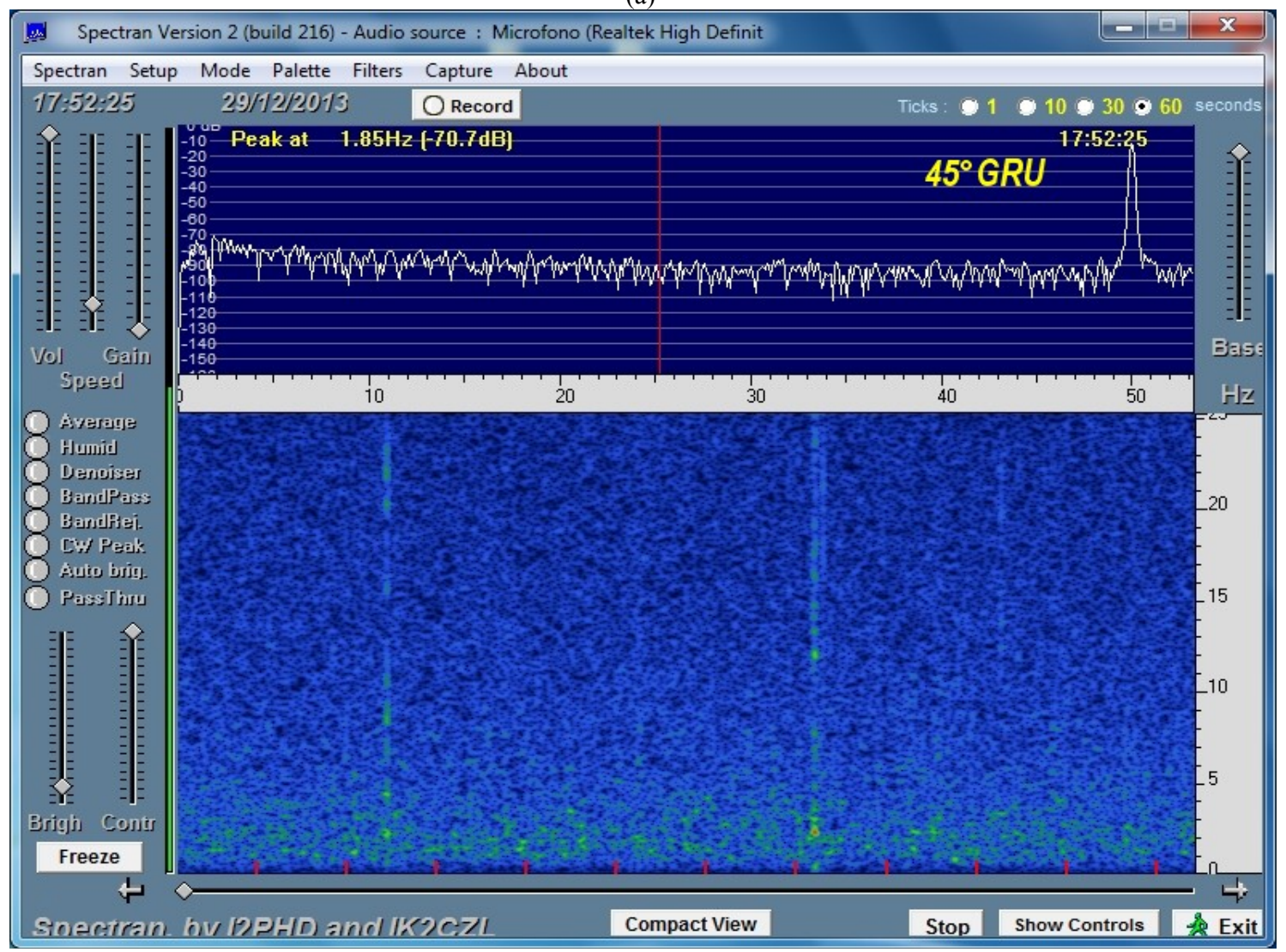

(b)

Fig. 5 Radio anomalies and acoustic measurements during the Matese earthquake of 29 December 2013.

Comparison of radio anomalies (a), measured in Rome by the Radio Emissions Project and acoustic emissions (b) measured in Rovigo by Dr. Jerry Ercolini, which preceded the Matese earthquake. The two spectrograms show the hourly levels of the electromagnetic and acoustic signals that proceed in more or less the same way up to the mainshock. 
The peak on the graph has a value of about 55 microseconds, but we know that this is a value averaged over 15 seconds, and we do not know if the instrument could record it at its maximum value.

4.2 Variations in the Geomagnetic and Gravitational Background Associated with Two Earthquakes in the Emilian Seism of May 2012

Reawakening of seismic activity in the Emilian Po Valley Plain (Italy) resulted in 2,492 earthquakes over five and a half months: 2,270 with $\mathrm{M}<3,189$ with a magnitude from $3.0=\mathrm{M}<4.0,27$ with $4.0<\mathrm{M}<5.0$, and 7 with $\mathrm{M}<6.0$. The mainshock was recorded during the night of 20 May 2012, at 04:03:52 Italian time (02:03:52 UTC) with epicentre in Finale Emilia, at a depth of $6.3 \mathrm{~km}$, by the INGV (Italian National Institute of Geophysics and Volcanology). A long sequence of telluric shocks occurred in the same seismic district in the area between the provinces of Modena, Ferrara, Mantua, Reggio Emilia, Bologna and Rovigo. In addition to the general devastation plus damage to civil and industrial buildings and historical heritage, the earthquake resulted in a total of 27 victims. Concomitant with the two strongest quakes, recorded on 20 and 29 May 2012, respectively, as in the case of others, variations were noted in the geomagnetic background by the LTPA monitoring station in Rome (Italy). These geomagnetic background variations were associated with the appearance of radio-anomalies in a frequency range from 0.1 to $3.0 \mathrm{~Hz}$, as well as gravimetric variations found around $60 \mathrm{~km}$ from the epicenter (Figs. 4a-4c). The peak accelerations, detected in correspondence with the strongest shocks on 20 and 29 May 2012, were respectively 0.31 and $0.29 \mathrm{~g}$.

The appearance of the radio-anomalies coincided, from a temporal point of view, with average gravimetric variations of approximately $30 \mu \mathrm{Gal}$ around the epicentre areas, concurrent with the main shock. In this example, both the appearance of radio anomalies and the gravitational variations recorded before strong earthquakes were related to the dynamics of the fault. The intense friction in the fault and the damping factors produced before the shock are hypothesized as being proportional to the number of radio-anomalies measured [23].

\subsection{Radio and Acoustic Emissions before the M5.2} Matese Earthquake (Italy) on 29 December 2013

On 29 December 2013, an earthquake of magnitude MW $=5.0$ (depth $10.5 \mathrm{~km}$ ) occurred in the Matese Mountain area at 18:08:43, local time. The earthquake was pinpointed by the INGV network to the Matese Mountains $\left(41.37^{\circ} \mathrm{N}, 14.45^{\circ} \mathrm{E}\right)$.

The areas closest to the epicentre suffered light damage to some buildings and places of worship. The most serious effects were seen in the cities of Piedimonte Matese and Faicchio, equal to VI-VII degrees of the MCS.

The VLF Monitor (Prototype Receiver $\mathrm{N}^{\circ} 1$ ) at the Radio Emissions Project monitoring station at Albano Laziale (Rome) recorded an intense radio emission that preceded the Italian M5.2 seism. This emission had a bandwidth of $3,700 \mathrm{~Hz}(13,800-17,500 \mathrm{~Hz})$ with a maximum peak around $15,658 \mathrm{~Hz}$. The main characteristic of this signal, as well as its high intensity, is that it features dozens of resonance harmonics spreading out from the main, more intense signal (at 15,658 Hz) gradually losing intensity.

The emission's bandwidth $(3,700 \mathrm{~Hz})$ was estimated considering also the position of the main signal's resonance harmonics and their intensity. These gradually lose intensity: at $\pm 1,850 \mathrm{~Hz}$ from the main signal reaching an intensity little higher than that of the natural background.

The VLF Monitor (Prototype Receiver $\mathrm{N}^{\circ}$ ) connected to another computer and a different antenna provided to the Radio Emissions Project, recorded the last 96 hours of natural electromagnetic background.

The results perfectly match the data recorded by Prototype Receiver $\mathrm{N}^{\circ} 1$. In fact, in this spectrogram we can observe the same electromagnetic anomaly 
centred on $15,658 \mathrm{~Hz}$ with the same bandwidth.

The monitoring of the radio anomalies showed an increase in the geomagnetic background about ten hours before the mainshock. At the same time, the acoustic signals accompanied the rise in the geomagnetic background, highlighted with vertical interference in the spectrogram. The earthquake occurred, in keeping with experience gained in the field, after a drop in electromagnetic and acoustic signals (Figs. 5a and 5b).

The appearance of acoustic emissions, identifiable in the graph with vertical lines, accompanied the increase in the geomagnetic background which preceded the M5.2 earthquake. Unlike the electromagnetic emissions, which diminished dramatically before and during the shock, in the case of the acoustic emissions, the peak coincided with the triggering of the seism.

\section{Conclusions}

The instrumentation proposed to measure the signals that precede and follow seisms on a global scale, which manifest more strongly as earthquake magnitude increases, can be put together for a modest cost and used in an educational lab. The individual experiences of Gabriele and Daniele Cataldi concerning radio anomalies, of Mario Campion in measuring gravity and Jerry Ercolini regarding acoustic emissions, show that synergies can exist between the signals, suitably interfaced with a computer, and using software that can be downloaded free of charge from the website. The experimental seismology project based on lab work and launched at the "Guglielmo Marconi" secondary school in Parma (Italy), has not yet collected enough data to produce statistics on the educational quality.

The method proposed, which is compatible with an "evidential paradigm", matches the "deterministic chaos" concept and, in the case of seismology, sets out to follow a natural phenomenon that will in any case occur, namely, the earthquake, but with variations which, from time to time, confirm that there are precise physical rules that "have not yet been written".

At present, therefore, it can only be stated that the working method is arousing an important element for the study of Natural Science and Seismology in the students: "curiosity".

\section{Acknowledgements}

I would like to express heartfelt thanks to Gabriele Cataldi, Daniele Cataldi, Mario Campion and Jerry Ercolini for their technical support during the educational experiment and their constant encouragement to pursue scientific research. Further thanks must go to Prof. Adriano Cappellini, Headmaster of the "G. Marconi”" secondary school for his help and support with the experimental seismology project.

\section{References}

[1] Gregori, G. P., Paparo, G., Poscolieri, M., and Zanini, A. 2005. "Acoustic Emission and Released Seismic Energy." Natural Hazards and Earth System Sciences 5: 777-82.

[2] Gregori, G. P., and Paparo, G. 2004. "Acoustic Emission (AE). A Diagnostic Tool for Environmental Sciences and for Non Destructive Tests (with a Potential Application to Gravitational Antennas)." In Meteorological and Geophysical Fluid Dynamics (A Book to Commemorate the Centenary of the Birth of Hans Ertel), edited by Schröder, W. Arbeitkreis Geschichte der Geophysik und Kosmische Physik, Science Edition, Bremen, 166-204.

[3] Paparo, G., Gregori, G. P., Coppa, U., De Rittis, R., and Taloni, A. 2002. "Acoustic Emission (AE) as a Diagnostic Tool in Geophysics." Annals Geophysics 45: 401-16.

[4] Freund, F. 2000. "Time-Resolved Study of Charge Generation and Propagation in Igneous Rocks.” Journal Geophysical Research 105 (B5): 11001-19.

[5] Freund, F. 2002. "Charge Generation and Propagation in Igneous Rocks.” Journal of Geodynamics 33 (4-5): 543-70.

[6] Takeuchi, A., Lau, B., and Freund, T. 2006. "Current and Surface Potential Induced by Stress-Activated Positive Holes in Igneous Rocks." Physics and Chemistry of the Earth Parts A/B/C. 31 (4-9): 240-7.

[7] Hayakawa, M., Itoh, T., and Smirnova, N. 1999. "Fractal Analysis of ULF Geomagnetic Data Associated with the Guam Earthquake on August 8, 1993." Geophys. Res. 
Lett. 26 (18): 2797-800.

[8] Hayakawa, M. 2011. "On the Fluctuation Spectra of Seismo-Electromagnetic Phenomena." Nat. Hazards Earth Syst. Sci. 11: 301-8.

[9] Hayakawa, M., Molchanov, O., Ondoh, T., and Kawai, E. 1996. "Anomalies in the Sub-ionospheric VLF Signals for the 1995 Hyogo-ken Nambu Earthquake." Journal of Physics of the Earth 44 (4): 413-8.

[10] Fraser-Smith, A. C., Bernardi, A., McGill, P. R., Ladd, M. E., Helliwell, R. A., and Villard, O. G. Jr. 1990. "Low-Frequency Magnetic Field Measurements near the Epicenter of the Ms 7.1 Loma Prieta Earthquake." Geophys. Res. Lett. 17: 1465-8.

[11] Straser, V. 2011. "Radio Anomalies, Ulf Geomagnetic Change and Variations in the Interplanetary Magnetic Field Preceding the Japanese M9.0 Earthquake." New Concepts in Global Tectonics Newsletter 59: 78-88.

[12] Straser, V. 2012. "Intervals of Pulsation of Diminishing Periods and Radio Anomalies Found before the Occurrence of M6+ Earthquakes." New Concepts in Global Tectonics Newsletter 65: 35-46.

[13] Straser, V. 2011. "Radio Anomalies and Variations in the Interplanetary Magnetic Field (IMF) Used as Seismic Precursors on a Global Scale." New Concepts in Global Tectonics Newsletter 61: 52-65.

[14] Straser, V. 2014. "Gravitational Anomalies and the Allais Effect Found in Italy during Eclipses and Strong Earthquakes." In Proceeding NPA 2014 Conference.

[15] Straser, V., Cataldi, G., and Cataldi, D. 2015. "Solar Wind Ionic Variation Associated with Earthquakes Greater than Magnitude 6.0." New Concepts in Global Tectonics Journal 3 (2): 140-54.

[16] Straser, V., Cataldi, G., and Cataldi, D. 2015. "Solar Wind Ionic and Geomagnetic Variations Preceding the M8.3 Chile Earthquake." New Concepts in Global
Tectonics Journal 3 (3): 394-9.

[17] Straser, V., and Cataldi, G. 2014. "Solar Wind Proton Density Increase and Geomagnetic Background Anomalies before Strong m6+ Earthquakes." In Proceedings of MSS-14, 280-6.

[18] Cataldi, G., Cataldi D., and Straser, V. 2013. "Variations of terrestrial Geomagnetic Activity Correlated to M6+ Global Seismic Activity." Geophysical Research Abstracts Vol. 15. EGU2013-2617, 2013, EGU General Assembly 2013.

[19] Mikhailov, V. O., Panet, I., Hayn, M., Timoshkina, E. P., Bonvalot, S., Lyakhovsky, V., Diament, M., and de Viron, O. 2014. "Comparative Study of Temporal Variations in the Earth'S Gravity Field Using GRACE Gravity Models in the Regions of Three Recent Giant Earthquakes." Izvestiya, Physics of the Solid Earth 50 (2): 177-91.

[20] Simonenko, S. V. 2014. "The Practical Forecasting Aspects of the Thermohydrogravidynamic Theory of the Global Seismotectonic Activity of the Earth Concerning to the Japanese Earthquakes near the Tokyo Region." American Journal of Earth Sciences 1 (2): 38-61.

[21] Straser, V. 2010. "Variations in Gravitational Field, Tidal Force, Electromagnetic Waves and Earthquakes." New Concepts in Global Tectonics Newsletter 57 (2010): 98-108.

[22] Zhu, Y., Fang, L., and Shusong, G. 2011. “Temporal Variation of Gravity Field before and after Wenchuan Ms8.0 Earthquake." Geodesy and Geodynamics 2 (2): 33-8.

[23] Straser, V. 2013. "Variations in the Geomagnetic and Gravitational Background Associated with Two Strong Earthquakes of the May 2012 Sequence in the Po Valley Plain (Italy)." Geophysical Research Abstracts, Vol. 15. EGU2013-1949, EGU General Assembly 2013. 